\title{
COMPARATIVE STUDY OF WAVELET \\ TRANSFORM AND WAVELET PACKET TRANSFORMATION IN IMAGE DENOISING USING THRESHOLDING TECHNIQUE
}

Ridhi Bhatnagar ${ }^{1}$, Aparna Vyas ${ }^{2}$

E-Mail Id: ridhibhatnagar89@gmail.com ${ }^{1}$, aparna@mru.edu.in²

Department of Mathematics, Manav Rachna University, Faridabad, India

\begin{abstract}
In this modern world where images are considered to be the mode of communication among people and the presence of noises in images can affect the information, which is present in that images, to retain back the original information wavelet transformation and wavelet packet transformation can be used. This paper deals with the comparative result of image denoising using Wavelet Transformation and Wavelet Packet transformation with gaussian noise using thresholding technique, in order to have look on the transformation that can provide the better result to get back the original information of images, the comparative result is measured by Peak signal to noise ratio (PSNR) and mean square value (MSE).
\end{abstract}

Keywords: Image denoising, wavelets, wavelet transformation, wavelet packet transformation.

\section{INTRODUCTION}

In this modern world, image is considered to be one of the mode of communication but due to nature or electronic devices error images are corrupted by various type of noises and to retain back the original information is known as image denoising. Noise can be of many type Gaussian, salt \& pepper, speckle.

Earlier Fourier analysis and short time Fourier transformation was considered to be an important tool for signal and image analysis, after the limitation that Fourier analysis that it cannot offer high frequency and time resolution at same time leads to the introduction of short time Fourier transformation but fails to provide the complete multi resolution analysis which leads to the formation of wavelets, which was introduced by Grossman and Morlet [1] in order to overcome multi resolution problem.

In recent years[4-9], many methods for image denoising came in limelight using wavelet transformation, Wavelet Packet Transformation, Curvelet transformation, Shearlet transformation or other multi resolution analysis tools, Donoho and stone [2] introduced the world to thresholding by coiling hard and soft thresholding or soft thresholding denoising method using wavelets, Malfait and Roose [3] denoise image using wavelet transformation by considering to measures novel and classic measure.

Wavelet packet transformation can be considered as the idea with general application that can produce the arbitrary resolution of wavelet tree decomposition in the $19^{\text {th }}$ century coif man and Wicker Hauser shower the light on the mathematical work of wavelet packet transformation.

In this paper we will compare the result obtained by wavelet transformation and wavelet packet transformation in order to have a look that which transformation provides us the better result for image denoising by comparing the peak signal to noise ratio and Mean square value of both.

\section{WAVELETS}

Wavelets was discovered by Grossman and Morlet [1], the main reason for the discovery of wavelet is because the Fourier analysis is totally dependent on the oscillating building blocks which is poorly suited or non-stationary signals. 
7 $\mathrm{F} \leqq$ International Journal of Technical Research \& Science

A function $\psi \in L^{2}(\mathbb{R})$ is called an orthonormal wavelet if the $\operatorname{system}\left\{\psi_{j, k}\right\}$ forms an orthonormal basis for $L^{2}(\mathbb{R})$ where $\psi_{\mathrm{j}, \mathrm{k}}=2^{\frac{\mathrm{j}}{2}}\left(2^{\mathrm{j}} \mathrm{x}-\mathrm{k}\right)$ where $\mathrm{j}, \mathrm{k} \in \mathbb{Z}$

\subsection{Wavelet Transformation}

The wavelet transform was first introduced in the context of a mathematical transform by Grossman and Morlet in 1984 [1]. It is a tool which allows us to cut the data, function or operator into different frequency which allow us to study each component with a resolution that can match to the scale.

\subsubsection{Continuous Wavelet Transformation}

The continuous wavelet transformation (CWT) of a function $f(x) \in L^{2}(\mathbb{R})$ with respect to $\psi \in L^{2}(\mathbb{R})$ is given by

$$
C W T(f)(a, b)=|a|^{\frac{j}{2}} \int_{-\infty}^{\infty} f(x) \psi\left(a^{j}(x-b)\right) d x
$$

Continuous wavelet transformation is considered to be very effective in determining the damping ration of the oscillating signal. It is considered to be better than Short time Fourier trans-formation which was developed after the determination of Fourier transformation. CWT is used in decomposing the signal into high and low frequency components. They consists of real valued and complex valued wavelet

\subsubsection{Discrete Wavelet Transformation}

The discrete wavelet transformation (DWT) of a given function $f(x) \in L^{2}(\mathbb{R})$ with respect to $\psi \in L^{2}(\mathbb{R})$ is given by

$$
\operatorname{DWT}(f)(a, b)=|a|^{\frac{j}{2}} \int_{-\infty}^{\infty} f(x) \psi\left(a_{0}^{j} x-k b_{0}\right) d x
$$

Where, $a_{0} \neq 0$ and $j, k \in \mathbb{Z}$. DWT transforms the discrete time signal to a discrete wavelet representation it is very beneficial in removing noises from image and it can also be termed as image processing. DWT is capable in various application fields such as from signal analysis to signal compression. Main application is to remove noise from an all kind of signals; images can be stated as image denoising. DWT decomposes the signal into 'approximation' and 'detail' coefficients at each level.

\section{THRESHOLDING}

Donoho and Johnson, they proposed the non-linear strategy for thresholding and introduce that thresholding can be applied in two ways either by hard thresholding or by soft thresholding, the value below which the details are considered small enough to be set to zero known as threshold.

Hard Thresholding: They set the coefficients value to zero whose value are less than $\lambda$. It is very much sensitive to small change in the signal.

Soft Thresholding: It is considered to be the extension of hard thresholding and it is suitable to coefficient value to zero whose value is lower than the threshold value. It is bias when the coefficients are large.

There are many approaches which deal to calculate the threshold value deals with the estimation of noise level. There are 3 main threshold selection algorithm rigorous, minimax, universal threshold estimation techniques that are mostly taken into consideration.

\subsection{Parameters}

There are many parameters to measure if the task is successful and 2 most common parameters are explained below:

\subsubsection{Mean Square Error (MSE)}

MSE or mean-square error can be simply explained as it is the subtraction of the estimated value of the image to the average value of the image and plays an important role in deter-mining the result in image denoising and 


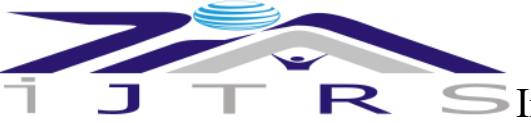

image compression that the result is satisfactory or not. For example, image is said to be denoised if the MSE value decreases and it can be determined as

$$
M S E=A-B
$$

Where $\mathrm{A}$ is the total number of bits of image and $\mathrm{B}$ is the total number of pixels present in images.

\subsubsection{Peak Signal to Noise Ratio (PSNR)}

It is one of the parameter to determine the result of the image is denoised or not by comparing the denoised image with the original image similarly noised image with the original image, image is said to be denoised if its PSNR value increases. PSNR vale of image can be calculated as

$$
P S N R=20 \log _{10} \frac{M}{\sqrt{M S E}}
$$

Where $\mathrm{M}$ is $\mathrm{M}$ is the maximum signal value that exists in the respective original image. And MSE is the Mean-square value.

\section{WAVELET PACKET TRANSFORMATION}

The main reason for the introduction of wavelet packet transformation is the limitation of wavelet transformation, Wavelets was introduced with many application in images and have a bright future for the smooth images but somehow fail to move this application in images which have high texture because texture rich images consists of small scale coefficient and these coefficient consists of little energy that is quantized to zero. Wavelet packet transformation was first discovered by coiffman and Wickerhauser in the 19thcentury the main reason for the discovery of wavelet packet transformation is because discrete wavelet transformation provide time-frequency localization but and fails to provide the information in the high-frequency region with low resolution in order to overcome the limitation in discrete wavelet trans-formation it leads to the introduction of wavelet packet transformation as it comes out to be capable to decompose a signal and provide the information of the respective signal in high frequency region. Wavelet packet transformation provide the information of low frequency sub-band and high frequency-sub-band as compared to wavelet transformation which only splits the signal in detail and approximation coefficient while in wavelet packet transformation splitting of detail and approximation coefficient is taken place and provide us the new way to encode a signal.

$$
x_{p}^{n, j}=\frac{1}{2^{j}} \int_{\mathbb{R}} x(t) \mu_{n}\left(\frac{p-t}{2^{j}}\right)
$$

\section{PROCEDURE}

To denoise the image by using wavelet transformation or wavelet packet transformation, the following steps are carried out as per algorithm shown in figure 1 . We need to first introduce the noise in the image, then we apply wavelet transformation or wavelet packet transformation to the image and after that we apply thresholding and finally, take inverse wavelet transformation or inverse wavelet packet transformation and get denoised image. Further, determine the PSNR and MSE value of denoised for the comparison of results. 

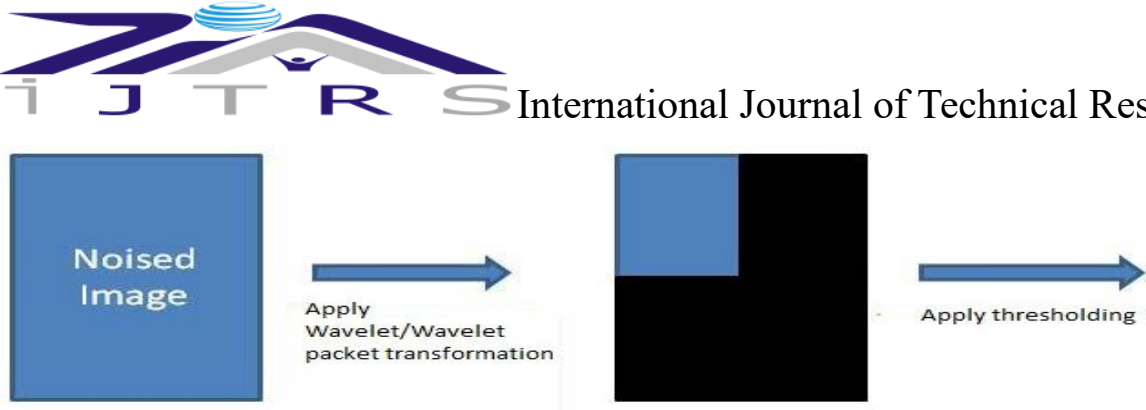

Apply thresholding
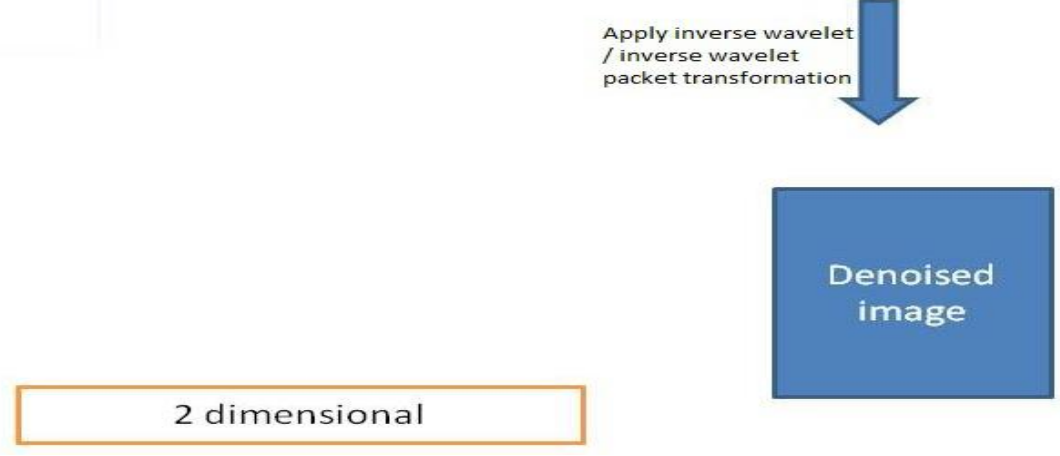

Fig 1: Block Diagram

\section{EXPERIMENT AND RESULT}

For the experimentation, we have taken balcony view image and we need to follow the procedure part. First we add the Gaussian noise in the image with variance 0.02 and noisy image is decomposed using wavelet packet transform and different wavelet transforms such as Haar wavelet, Coiflet wavelet, Daubechies wavelet and Symlet wavelet. First level or second level decomposition is applied to the noisy image. We have performed Soft and Hard Thresholding at each decomposition level of an image for wavelet packet transform and different wavelet transforms. At each level the value of thresh-old is different for example at the end the inverse of the wavelet packet trans-form and wavelet transform has been performed to get the denoised image. as shown in fig. 6.1. The performance of various wavelet transforms has been compared with the help of various parameters such as Peak signal to Noise Ratio (PSNR) and Mean Square Error (MSE). We can easily see from the results that wavelet packet transform gives better performance in comparison to wavelet transform.

The below image is denoised, having the PSNR value of the below image is 20.6095 and MSE 365.3605.

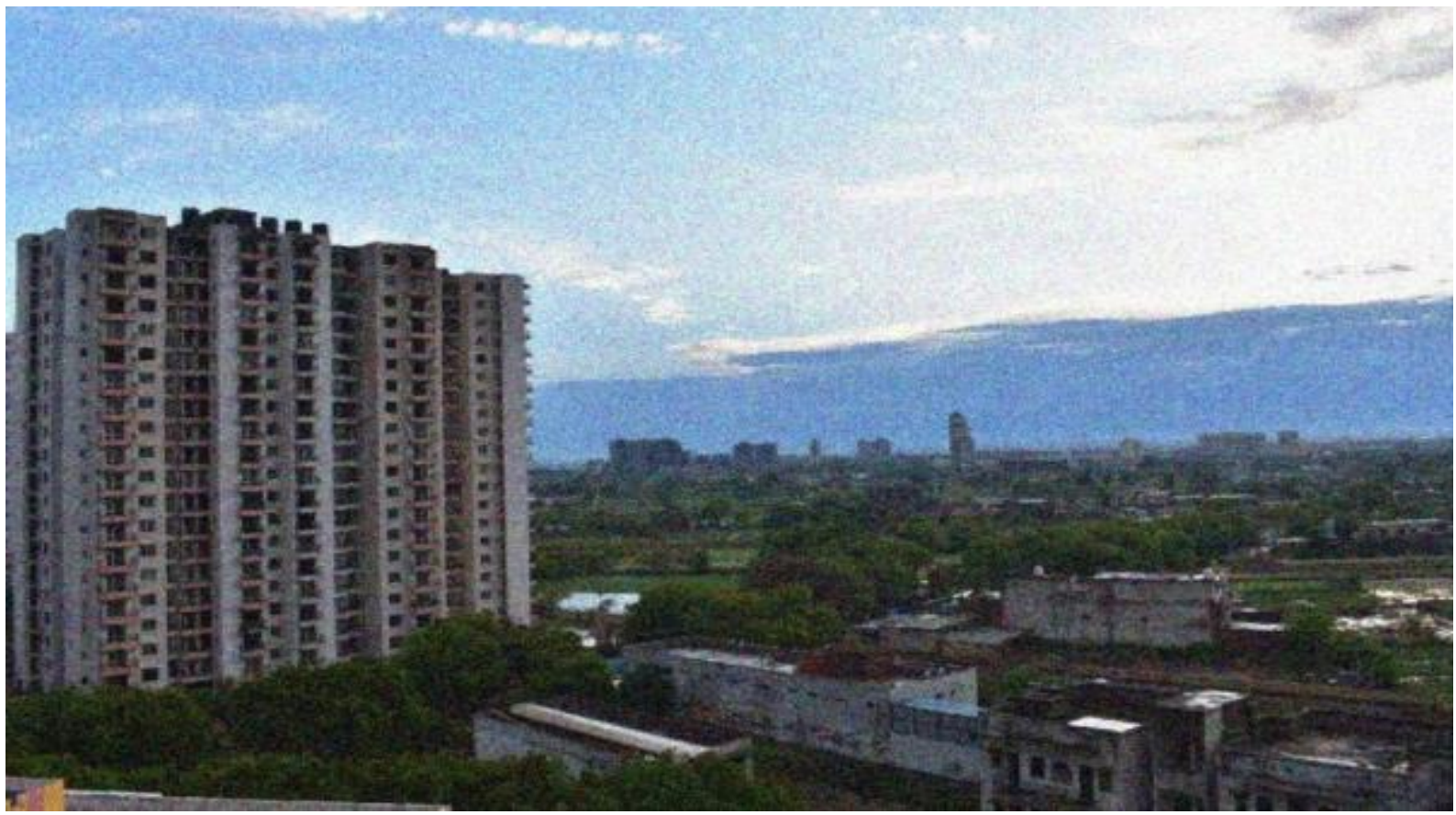

Fig. 6.1 Denoised Image

DOI Number: https://doi.org/10.30780/IJTRS.V06.I01.002

pg. 13

Paper Id: IJTRS-V6-I1-003

www.ijtrs.com, www.ijtrs.org

Volume VI Issue I, January 2021 @2017, IJTRS All Right Reserved 
T $\mathrm{I} \leftrightarrows$ International Journal of Technical Research \& Science

Table- 6.1 Experimental Result

\begin{tabular}{|c|c|c|c|c|}
\hline \multirow{2}{*}{$\begin{array}{l}\text { Denoised } \\
\text { using }\end{array}$} & \multicolumn{2}{|c|}{ Wavelet Transformation } & \multicolumn{2}{|c|}{ Wavelet Packet Transformation } \\
\hline & Denoised image & $\begin{array}{l}\text { PSNR and } \\
\text { MSE }\end{array}$ & Denoised Image & PSNR and MSE \\
\hline $\begin{array}{l}\text { Haar } \\
\text { wavelet }\end{array}$ & & $\begin{array}{l}\text { PSNR=21.481 } \\
2 \\
\text { MSE }=270.62 \\
7\end{array}$ & & $\begin{array}{l}\text { PSNR }=22.3816 \\
M S E=248.262\end{array}$ \\
\hline $\begin{array}{l}\text { Coiflet } \\
\text { wavelet }\end{array}$ & & $\begin{array}{l}\text { PSNR=20.975 } \\
2 \\
\text { MSE }=300.41 \\
6\end{array}$ & & $\begin{array}{l}P S N R=21.7635 \\
M S E=279.762\end{array}$ \\
\hline
\end{tabular}

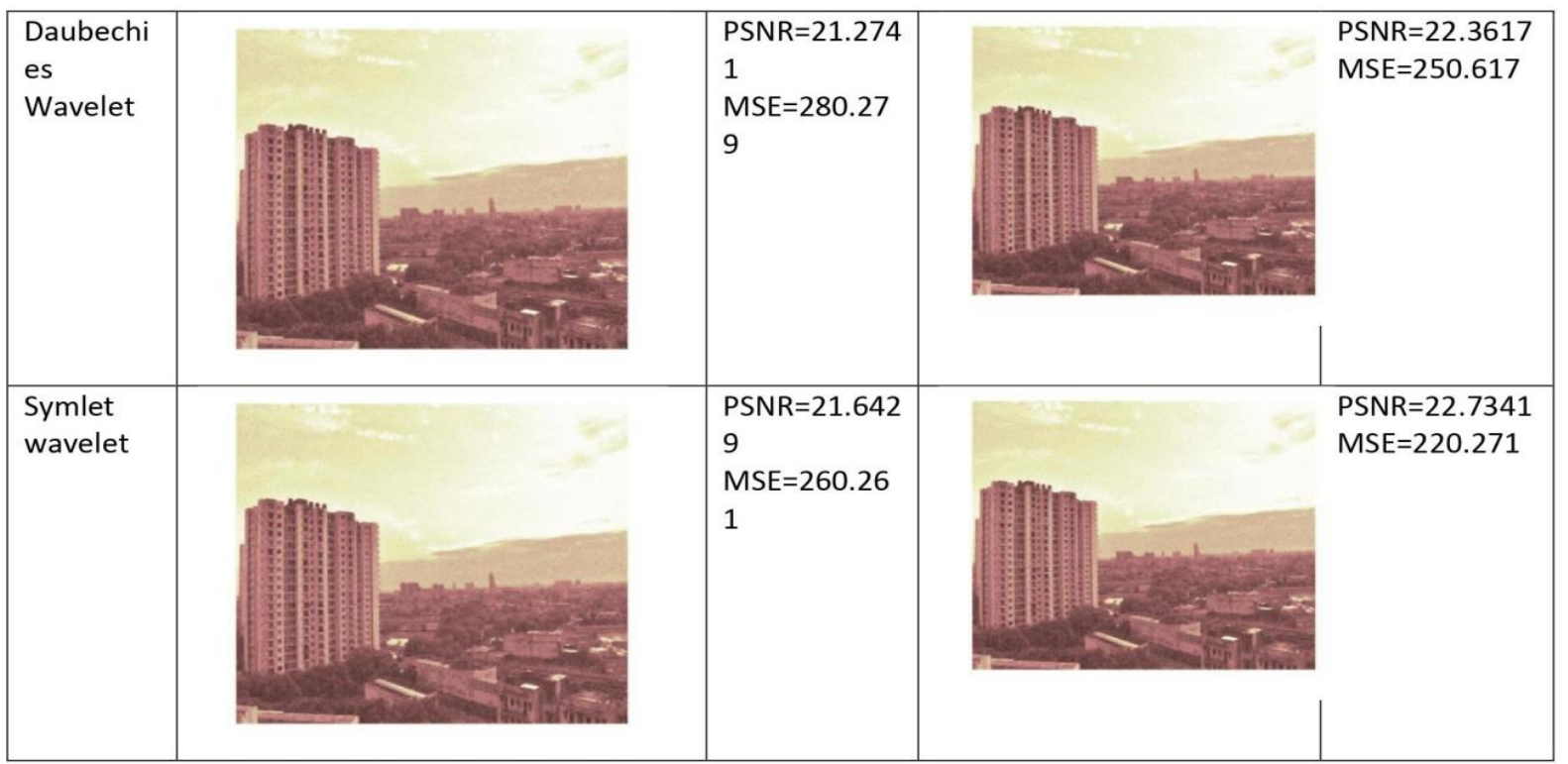

\section{CONCLUSION}

This paper describes that comparative result of image denoising using wavelet and wavelet packet transformation (Coiflet, Haar, Daubechies, Symlet wavelet) and in all the given result it was found that the images denoised using wavelet packet transformation shows more desirable result as compared to the wavelet transformation when measured from the parameter PSNR and MSE.

\section{REFERENCES}

[1] A. Grossmann and J. Morlet, "Decomposition of Hardy functions into square integrable wavelets of constant shape”, SIAM J. Anal. 15, pp. 723-736, 1984.

[2] D. L Donoho and I. M. John stone, "Ideal spatial adaptation via wavelet shrinkage", Biometrika, 81 (3) 425-455, 1994.

DOI Number: https://doi.org/10.30780/IJTRS.V06.I01.002 
ᄀ $\longrightarrow$ \& International Journal of Technical Research \& Science

[3] M. Malfait and D.Roose, "Wavelet based image denoising using a Markov random field a priori model", IEEE Trans. Image Process. 6(4) (1997).

[4] Ankita Yadav, Riya Fagna and Aparna Vyas, "Image Denoising using wavelet Transform and Shearlet Transform", published in conference proceedings Middle East International Conference On contemporary scientific studies-IV, Beirut, Lebanon, Turkey November 1-3,2020, ISBN No.: 978-625-7279-29-1.

[5] Ridhima Nehra, Rojin Joseph and Aparna Vyas "Comparison of Image Denoising Using Wavelet Transform and Wavelet Packet Transform" published in conference proceedings Tokyo Summit II 2nd International Conference on Innovative Studies of Contemporary Sciences, Tokyo, Japan, pp. 108-115, August 2020, ISBN No. 978-625-7139-21-2.

[6] Ridhi Bhatnagar and Aparna Vyas, "Image Processing using Multiscale Transform: A Review" published in conference proceedings Tokyo Summit II: 2nd International Conference on Innovative Studies of Contemporary Sciences, Tokyo, Japan, pp. 108-115, August 2020, ISBN No. 978-625-7139-21-2.

[7] Aparna Vyas and Joonki Paik, "Applications of Multiscale Transforms to Image Denoising: Survey" proceedings of International Conference on Electronics, Informations and Communication", IEEE Explore, Hawaii, USA, January 24-27, 2018.

[8] Aparna Vyas and Joonki Paik, "Review of the Application of Wavelet Theory to Image Processing", IEIE Transactions on Smart Processing and Computing, Vol. 5, No. 6,403-417, (KSCI, Scopus Index), eISSN:2287-5255,2016.

[9] Aparna Vyas, Soohwan Yu and Joonki Paik, "Multiscale Transforms with Application to Image Processing”, Springer Nature, Singapore, Pte Ltd., DOI:10.1007/978-981-10-7272-7, eBook ISBN: 978-981-10-7272-7 Hard-cover ISBN: 978-981-10-7271-0, Series ISSN: 1860-4862, PP: XVI, 345, Edition No.: 1, Jan, 2018. 